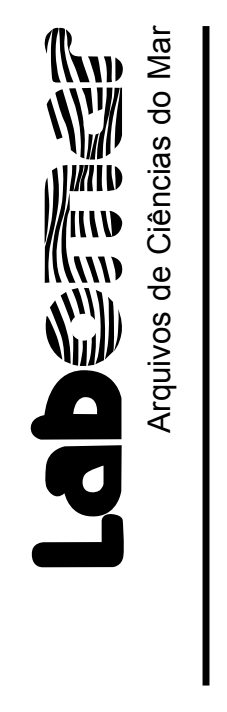

NOTA CIENTÍFICA

\title{
NOTAS ADICIONAIS SOBRE Diaphus adenomuS GILBERT, 1905 (ACTINOPTERYGII: MYCTOPHIDAE) NO ESTADO DA BAHIA, OCEANO ATLÂNTICO OCIDENTAL
}

\author{
Additional notes on Diaphus adenomus Gilbert, 1905 \\ (Actinopterygii: Myctophidae) in Bahia State, Brazil, western \\ Atlantic Ocean
}

Jailza Tavares de Oliveira-Silva1, Paulo Roberto Duarte Lopes ${ }^{2}$, George Olavo

\begin{abstract}
RESUMO
São apresentadas novas informações sobre caracteres merísticos e morfométricos e dieta alimentar com base em 40 indivíduos de Diaphus adenomus Gilbert, 1905 (Actinopterygii: Myctophidae), coletados no Estado da Bahia, Brasil, Oceano Atlântico ocidental. O comprimento total variou de 139,0 mm a 230,0 mm. Pequenas variações foram observadas com relação aos caracteres morfométricos, merísticos e proporções corporais. Maturidade sexual foi observada em indivíduos com comprimento variando entre $174,0 \mathrm{~mm}$ e 230,0 mm. A dieta alimentar de D. adenomus consiste principalmente de peixes e camarões.
\end{abstract}

Palavras-chaves: Diaphus adenomus, caracteres merísticos, caracteres morfométricos, dieta alimentar, Estado da Bahia.

\begin{abstract}
New information on meristic and morphometric characters and on feeding diet of Diaphus adenomus Gilbert, 1905 (Actinopterygii: Myctophidae), based on the catch of 40 specimens off Bahia State, Brazil, western Atlantic Ocean, is presented. Total length ranged from $139.0 \mathrm{~mm}$ to $230.0 \mathrm{~mm}$. Slight variations were observed with respect to meristic and morphometric characters and body proportions. Sexual maturity was observed in female specimens with length varying from $174.0 \mathrm{~mm}$ to $0230.0 \mathrm{~mm}$. The feeding diet $\mathrm{D}$. adenomus consists mainly of fish and shrimp.
\end{abstract}

Keywords: Diaphus adenomus, meristic character, morphometric character, feeding diet, Bahia State.

\footnotetext{
${ }^{1}$ Bióloga, Universidade Estadual de Feira de Santana, BR-116, km 03, Feira de Santana, BA 44036-900. E-mail: jtosilva@yahoo.com.br

2 Professor Assistente, Universidade Estadual de Feira de Santana, BR-116, km 03, Feira de Santana, BA 44036-900. E-mail: andariIho40@gmail.com

${ }^{3}$ Professor Assistente, Universidade Estadual de Feira de Santana, BR-116, km 03, Feira de Santana, BA 44036-900. E-mail: georgeolavo@uol.com.br
} 


\section{INTRODUÇÃO}

A família Myctophidae (ordem Myctophiformes) é marinha, ocorre do Ártico à Antártida e está composta por 33 gêneros e, no mínimo, 240 espécies. Estas são de hábito pelágico de mar aberto e encontradas principalmente na faixa dos $1.000 \mathrm{~m} \mathrm{su}$ periores da coluna d'água (mesopelágicas), poucas vivendo abaixo dessa faixa (batipelágicas) e algumas estão associadas aos taludes de ilhas e de continentes (pseudoceânicas) (Craddock \& Hartel, 2002; Nelson, 2006; Braga et al., 2014).

Diaphus adenomus Gilbert, 1905 (Figura 1), pertencente a esta família, distribui-se nas águas tropicais dos oceanos Atlântico e Pacífico. No Atlântico ocidental, está citada para o Golfo do México, Bahamas, Mar do Caribe, Nicarágua e Estado da Bahia (litoral nordeste do Brasil). Durante o dia, sua distribuição batimétrica varia de 500 a 600 $\mathrm{m}$ de profundidade enquanto à noite situa-se em 180 m sendo que a maioria de suas capturas são próximas ao fundo. Atinge pelo menos $24,6 \mathrm{~cm}$ de comprimento total, é considerada rara e é definida como sendo pseudoceânica (Nafpaktitis, 1974; McEachran \& Fechhelm, 1998; Craddock \& Hartel, 2002; Braga et al., 2008).

\section{MATERIAL E MÉTODOS}

Os exemplares aqui examinados encontram-se conservados em álcool 70\% e estão depositados na coleção da Divisão de Peixes do Museu de Zoologia, Universidade Estadual de Feira de Santana, sob número LIUEFS 15862, tendo sido coletados com rede-de-arrasto de fundo pelo N.Oc. Thalassa durante a Operação Bahia-2, na posição aproximada de $013^{\circ} 27^{\prime} \mathrm{S}-038^{\circ} 39^{\prime} \mathrm{W}$, a $1.106 \mathrm{~m}$ de profundidade.

Medidas biométricas foram obtidas com paquímetro (exceto o comprimento total -CT, obtido com ictiômetro e régua) e estão baseadas em Braga et al. (2008). Os exemplares foram dissecados para exame das gônadas para identificação do sexo e de possível estágio de maturação através de exame direto das gônadas e retirada do tubo digestivo para análise do seu conteúdo sob microscópio estereoscópico. O grau de repleção dos estômagos foi classificado visualmente em cheio, meio cheio, pouco cheio e vazio.

\section{RESULTADOS}

Foram examinados 40 exemplares de $D$. adenomus medindo entre $139,0 \mathrm{~mm}$ e $230,0 \mathrm{~mm}$ CT (entre $122,0 \mathrm{~mm}$ e $194,0 \mathrm{~mm}$ de comprimento padrão).

Um total de 38 fêmeas $(95,0 \%)$ e 2 machos $(5,0 \%)$ foram identificados. Entre as fêmeas, 55,0\% não estavam maduras (entre $139,0 \mathrm{~mm}$ e $195,0 \mathrm{~mm}$ CT), 35,0\% estavam maduras (entre $174,0 \mathrm{~mm}$ e 230,0 mm CT) e 10,0\% estavam em maturação. Os machos (totalizando 10,0\%) mediram $166,0 \mathrm{~mm}$ e $174,0 \mathrm{~mm}$ de CT e não estavam maduros.

Quanto ao grau de repleção, 77,5\% dos estômagos estavam pouco cheios enquanto $22,5 \%$ encontravam-se meio cheio. Já com relação ao grau de digestão, em 92,5\% dos estômagos o alimento encontrava-se digerido, enquanto em $7,5 \%$ estava meio digerido.

$\mathrm{O}$ volume de alimento ingerido variou entre menos de $0,1 \mathrm{ml}(27,5 \%)$ a $0,9 \mathrm{ml}(2,5 \%)$, com predominância dos valores de $0,1 \mathrm{ml}(42,5 \%), 0,2 \mathrm{ml}(17,5 \%)$ e $0,3 \mathrm{ml}(10,0 \%)$.

Com relação à composição da dieta, foram identificadas três categorias ali mentares.

Em freqüência de ocorrência predominaram os peixes (Actinopterygii Teleostei) observados em $67,5 \%$ dos estômagos e, em seguida, os camarões (Crustacea Decapoda Dendrobranchiata) e restos de crustáceos, numa proporção de 5,0\%. A única escama de Teleostei identificada foi considerada como parte de algum peixe ingerido (Tabela I).

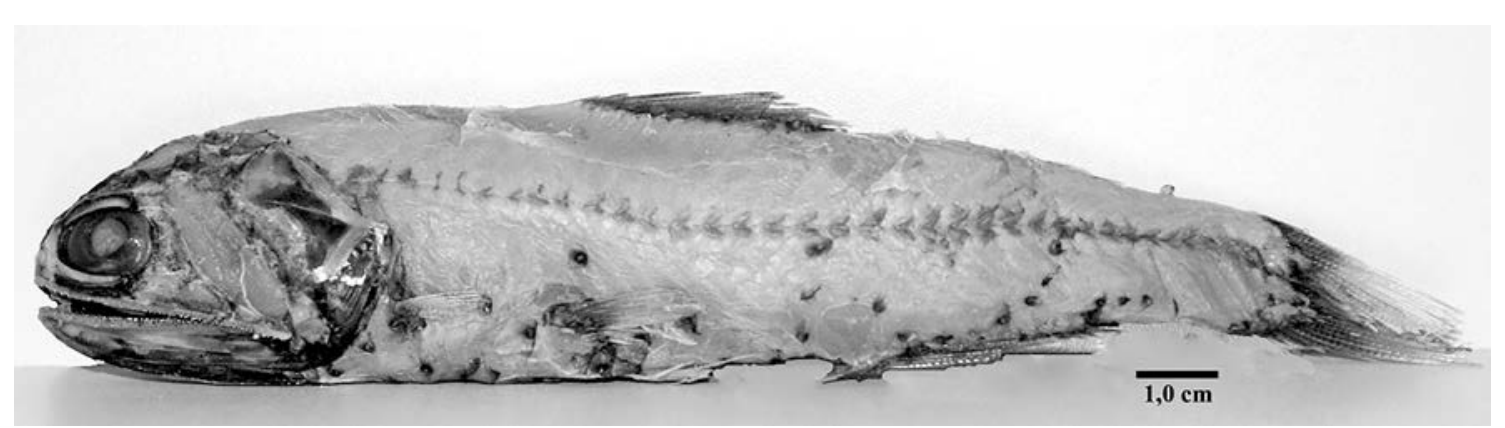

Figura 1 - Diaphus adenomus Gilbert, 1905 (LIUEFS 15862). 
Tabela I - Itens alimentares, e freqüência de ocorrência (FO) e frequência numérica (FN) em 40 estômagos de Diaphus adenomus.

\begin{tabular}{l|c|c}
\hline \multicolumn{1}{c|}{ Item alimentar } & FO (\%) & FN (\%) \\
\hline Actinopterygii Teleostei & 67,5 & 85,3 \\
Crustacea Decapoda Dendrobranchiata & 5,0 & 5,9 \\
Restos Crustácea & 5,0 & 5,9 \\
Escama Teleostei & 2,5 & 2,9 \\
Matéria orgânica digerida & 25,0 & --- \\
\hline
\end{tabular}

Em freqüência númerica, verificou-se um amplo predomínio de peixes $(85,3 \%)$ seguido por camarões e restos de crustáceos (camarões?), ambos com $5,9 \%$.

Os peixes ingeridos não puderam ser identificados devido ao elevado grau de digestão. No interior da boca de três exemplares de $D$. adenomus foram encontrados também Myctophidae, que provavelmente foram engolidos durante a subida da rede.

Todos os exemplares apresentavam vários Nematoda, parasitas no interior da cavidade abdominal.

\section{DISCUSSÃO}

Diaphus Eigenmann \& Eigenmann, 1890 é o gênero com maior número (60) de espécies conhecidas de Myctophidae. Santos \& Figueiredo (2008) e Menezes (2003) citam 18 espécies de Diaphus para a costa brasileira mas não incluem $D$. adenomus, que é citada pela primeira vez para o Brasil por Braga et al. (2008), ampliando esse número para 19.

A maioria das espécies de Diaphus ocorrentes no Brasil são de pequeno porte, atingindo comprimento inferior a 100,0 mm (Santos \& Figueiredo, 2008).

Braga et al. (2014) identificaram 29 espécies de Myctophidae (sendo 12 do gênero Diaphus incluindo D. adenomus) para a região da Zona Econômica Exclusiva brasileira entre $11^{\circ} \mathrm{S}$ e $22^{\circ} \mathrm{S}$. D. adenomus foi, em número de indivíduos, a quinta espécie mais capturada de Diaphus e a oitava no total de espécies de Myctophidae.

Algumas variações nos caracteres merísticos e proporções corporais foram observadas neste estudo quando comparados aos de exemplares examinados por Braga et al. (2008), ao largo da Bahia, contribuindo para um melhor conhecimento sobre $D$. adenomus ao longo de sua área de dis- tribuição geográfica (Tabela II). Quanto ao material examinado por Nafpaktitis (1974), apenas o número de escamas da linha lateral variou com relação ao material aqui examinado.

Os ciclos de vida de muitas espécies de Myctophidae são pouco conhecidos, especialmente aquelas que alcançam mais de $100,0 \mathrm{~mm}$ de comprimento (Craddock \& Hartel, 2002), como é o caso de D. adenomus. Portanto, as informações aqui apresentadas com relação a reprodução e alimentação contribuem para um melhor conhecimento da biologia desta espécie.

Os membros de Myctophidae se alimentam de copépodes, anfípodas, ostracodas, eufasiáceos, quetognatas, ovos e larvas de peixes (Hulley, 1986; Mceachran \& Fechhelm, 1998; Santos \& Figueiredo, 2008), sendo estas as presas de maior tamanho.

Segundo Tyler Jr. \& Pearcy (1975), ao largo da costa do Oregon (costa ocidental dos Estados Unidos), três espécies de Myctophidae são consideradas comuns, D. theta, Stenobrachius leucopsarus e Tarletonbeania crenularis, $e$ apresentam hábitos alimentares similares entre si, ingerindo principalmente eufasiáceos, copépodos e anfípodas, mas incluindo também ovos, pterópodos, ostracódas, poliquetas, quetognatos, salpas, peixes e diatomáceas, presas que em geral também apresentam um tamanho menor do que aquelas ingeridas por D. adenomus.

Com base nos dados aqui apresentados, embora preliminares devido à pequena amostra examinada, $D$. adenomus pode ser caracterizado como uma espécie predadora, principalmente sobre peixes, e que complementa sua dieta com crustáceos.

O aproveitamento de D. adenomus como possível recurso pesqueiro (devido ao seu tamanho,

Tabela II - Faixa de variação e média (x) dos caracteres morfométricos (mm) e merísticos, e proporções corporais de 40 exemplares de Diaphus adenomus.

\begin{tabular}{|c|c|c|}
\hline Caracteres & Presente trabalho & $\begin{array}{c}\text { Braga et al. } \\
(2008)\end{array}$ \\
\hline Número de espécimes & 40 & 136 \\
\hline Comprimento total (CT) & $139-230(\bar{x}=182,7)$ & $99-246$ \\
\hline Comprimento padrão (CP) & $122-194(\bar{x}=154,2)$ & 83-203 \\
\hline Nadadeira dorsal (ND) & $13-16(\bar{x}=14,17)$ & $15(14)$ \\
\hline Nadadeira anal (NA) & $13-16(\bar{x}=14)$ & $14(16)$ \\
\hline Nadadeiras peitorais (NP) & 8 (danificado) $-12(x=12)$ & $12(11)$ \\
\hline Rastros branquiais & $5(6)(\bar{x}=5)+1+10(11)(\bar{x}=11)$ & $5+1+11$ \\
\hline Escamas da linha lateral & $31-36$ (danificada) $(\bar{x}=33)$ & 37 \\
\hline Relação CC/CP (\%) & $22,67-33,19(\bar{x}=28,81)$ & $26,6-30,0$ \\
\hline Relação CC/CT $\mathrm{max}_{\max }(\%)$ & $1,09-1,97(x=1,35)$ & $1,2-1,6$ \\
\hline $\mathrm{CT}_{\max } / \mathrm{DO}(\%)$ & $1,89-4,39(x=2,67)$ & $2,0-3,1$ \\
\hline CC /DO (\%) & $2,51-5,93(x=3,58)$ & $3,0-4,3$ \\
\hline
\end{tabular}


maior do que a maioria das espécies de Myctophidae) depende de maiores informações sobre sua biologia, bem como sobre o papel exercido pelos Nematoda parasitas encontrados na cavidade abdominal.

Agradecimentos - À coordenação do Programa REVIZEE/SCORE Central e ao Prof. Dr. Gustavo W. Nunan (UFRJ, MNRJ) (†) pela liberação do material aqui analisado para incorporação na coleção de peixes do Museu de Zoologia da UEFS.; à Bahia Pesca S.A. e à UEFS pelo apoio logístico no embarque do segundo e terceiro autores no N.Oc. Thalassa, e incorporação do material coletado; à tripulação desde navio de pesquisa, pelo auxílio a bordo.

\section{REFERÊNCIAS BIBLIOGRÁFICAS}

Braga, A.C.; Costa, P.A.S.; Lima, A.T.; Nunan, G.W.; Olavo, G. \& Martins, A.S. Padrões de distribuição de teleósteos epi- e mesopelágicos na costa central (11$22^{\circ} \mathrm{S}$ ) brasileira, p.63-86, in Costa, P.A.S., Olavo, G. \& Martins, A.S. (eds.), Biodiversidade da fauna marinha profunda na costa central brasileira. Museu Nacional (Série Livros n. 24), 184 p., Rio de Janeiro, 2007.

Braga, A.C.; Costa, P.A.S. \& Nunan, G.W. First record of the firebrow lanternfish Diaphus adenomus (Myctophiformes: Myctophidae) from the South Atlantic. J. Fish. Biol., v.73, p.296-301, 2008.

Braga, A.C.; Costa, P.A.S.; Martins, A.S.; Olavo, G. \& Nunan, G.W. Lanternfish (Myctophidae) from eastern Brazil, southwest Atlantic Ocean. Lat. Am. J. Aquat. Res., v.42, n.1, p.245-257, 2014.
Craddock, J.E. \& Hartel, K.E. Myctophidae, p. 944951, in Carpenter, K. (ed.), The living marine resources of the Western Central Atlantic. Volume 2: Bony fishesPart 1 (Acipenseridae to Grammatidae). FAO Species Identification Guide for Fishery Purposes and American Society of Ichthyologists and Herpetologists Special Publication, n.5, Rome, 2002.

Hulley, P.A. Family no. 86: Myctophidae, p. 282-321, in Smith, M.M. \& Heemstra, P.C. (eds.), Smiths' sea fishes. Springer-Verlag, 1047 p., Berlin, 1986.

McEachran, J.D. \& Fechhelm, J.D. Fishes of the Gulf of Mexico. Vol.1: Myxiniformes to Gasterosteiformes. University of Texas, 1112 p., Austin, 1998.

Nafpaktitis, B.G. A new record and a new species of lanternfish, genus Diaphus (family Myctophidae), from the north Atlantic Ocean. Contrib. Sci., n.254, p.1-6, 1974.

Nelson, J.S. Fishes of the world. John Wiley \& Sons, $4^{\text {th }}$ edition, 601 p., New Jersey, 2006.

Santos, A.P. \& Figueiredo, J.L. Guia de identificação dos peixes da família Myctophidae do Brasil. Museu de Zoologia da Universidade de São Paulo, 168 p., São Paulo, 2008

Santos, A.P.; Figueiredo, J.L. \& Menezes, N.A. Família Myctophidae, p.52-57, in Menezes, N.A., Buckup, P.A., Figueiredo, J.L. \& Moura, R.L. (eds.), Catálogo das espécies de peixes marinhos do Brasil. Museu de Zoologia da Universidade de São Paulo, 159 p., São Paulo, 2003.

Tyler Jr., H.R. \& Pearcy, W.G. The feeding habits of three species of lanternfishes (family Myctophidae) off Oregon, USA. Mar. Biol., v.32, p.7-11, 1975. 